\section{ELIMINATING BIOFILM}

Nuview has launched the latest product in its Continu range, the Dental Unit Water Line Disinfectant.

Unlike alcohol or bleach-based products, Continu is highly effective at eliminating biofilm to achieve long-term reduction in microbial contamination yet, being water based, it is very gentle making it harmless to water lines and safe for patients. It is also economical compared to existing solutions.

The new Dental Unit Water Line Disinfectant completes the range that includes spray, wipes, liquid soap and hand cleansing foam, together with an Anti Microbial Dental Impression Mix. Reader response number 54

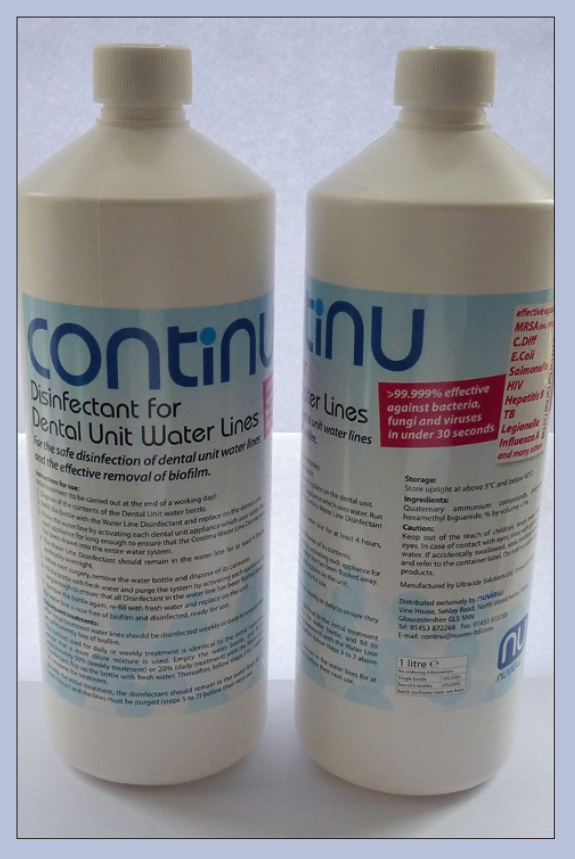

Without resorting to alcohol or aldehydes, Saniswiss biosanitizer digests viruses, bacteria and fungi and converts such germs to water utilising a unique and patented super oxygenised solution.

When Saniswiss biosanitizer is applied to the germs the electrically charged disinfectant enters the cells causing an electrical exchange.

Saniswiss biosanitizer oxidizes the cells causing instant death to the virus, bacteria or fungi, ensuring complete disinfection without any of the inherent risks associated with alcohol or aldehyde-based products.

Being a true water-based product, Saniswiss biosanitizer is in contact with the surface for longer - it will not evaporate in seconds like alcohol, meaning it is even more effective at killing germs. Reader response number 55

\section{AUTOMATIC CALIBRATION FACILITY}

Quality Endodontic Distributors Ltd, UK distributors of the Morita ZX apex locator, have recently introduced the new Root ZX Mini.

Compact and lightweight, the Root ZX Mini facilitates accurate measurement of the root canal, requires no zero adjustment and incorporates an automatic calibration facility and a clear liquid crystal display. It supplements the existing Dentaport System and the Tri Auto ZX.

The compact Root ZX Mini apex locator is battery operated, light, tough and easily transported. Featuring a reflector-type colour liquid crystal screen and piezoelectric beeper it makes accurate measurements whether the canal is dry or wet. File position and movement are clearly shown in real time with easy to read increments for high precision measurements.

The display instantly changes to green as the file approaches the apex. File position is also indicated by the audible signal for additional precision. Easy to use, the Root ZX Mini can memorise two types of working length.

\section{Reader response number 56}

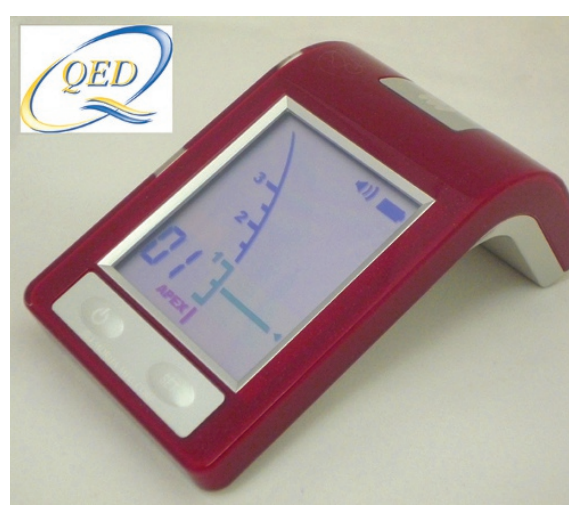

\section{EXPANDED RANGE} OF BODY SHADES

Filtek Supreme XTE universal restorative from 3M ESPE is the latest innovation in the Filtek line of restoratives. With the same physical properties of Filtek Supreme XT universal restorative, it now brings an expanded range of body shades. This delivers more universal options for creating easy, natural- looking, one-shade restorations, while maintaining the composite's versatility for dual or multi-layering techniques.

This unique technology allows further advancements including improved handling of translucent shades, as well as enhanced fluorescence and better polish retention with dentine, enamel and body opacities. New bold labelling and colour-coded opacities simplify the shade selection process. Additionally, translucent shades are now available in capsule delivery.

As the first and only composite system to incorporate true nanotechnology, the Filtek Supreme restorative line has gained a strong following over the past six years, popular for its ability to provide the polish and retention of a microfill, while maintaining the strength and wear properties of a modern hybrid. Reader response number 57 